\title{
Energy-Spectral Efficiency Optimization in Wireless Underground Sensor Networks Using Salp Swarm Algorithm
}

\author{
Mariem Ayedi $\mathbb{D}^{1,2}$ Esraa Eldesouky $\mathbb{D}^{1,},{ }^{1,3}$ and Jabeen Nazeer $\mathbb{D}^{1}$ \\ ${ }^{1}$ Department of Computer Science, College of Computer Engineering and Sciences, Prince Sattam Bin Abdulaziz University, \\ Al-kharj 11942, Saudi Arabia \\ ${ }^{2}$ MEDIATRON Lab., SUP'COM, Carthage University, Tunis 2083, Tunisia \\ ${ }^{3}$ Department of Computer Science, Faculty of Computers and Informatics, Suez Canal University, Ismailia 41522, Egypt
}

Correspondence should be addressed to Mariem Ayedi; m.ayedi@psau.edu.sa

Received 25 October 2020; Revised 12 December 2020; Accepted 2 January 2021; Published 15 January 2021

Academic Editor: Jesús Lozano

Copyright (C) 2021 Mariem Ayedi et al. This is an open access article distributed under the Creative Commons Attribution License, which permits unrestricted use, distribution, and reproduction in any medium, provided the original work is properly cited.

Achieving high data rate transmission is critically constrained by green communication metrics in Wireless Sensor Networks (WSNs). A unified metric ensuring a successful compromise between the energy efficiency (EE) and the spectral efficiency (SE) is, then, an interesting design criterion in such systems. In this paper, we focus on EE-SE tradeoff optimization in Wireless Underground Sensor Networks (WUSNs) where signals penetrate through a challenging lossy soil medium and nodes' power supply is critical. Underground sensor nodes gather and send sensory information to underground relay nodes which amplifyand-retransmit received signals to an aboveground sink node. We propose to optimize source and relay powers used for each packet transmission using an efficient recent metaheuristic optimization algorithm called Salp Swarm Algorithm (SSA). Thus, the optimal source and relay transmission powers, which maximize the EE-SE tradeoff under the maximum allowed transmission powers and the initial battery capacity constraints, are obtained. Further, we study the case where the underground medium properties are dynamic and change from a transmission to another. For this situation, we propose to allocate different maximum node powers according to the soil medium conditions. Simulation results prove that our proposed optimization achieves a significant EE-SE tradeoff and prolongs the network's lifetime compared to the fixed allocation node power scheme. Additional gain is obtained in case of dynamic medium conditions.

\section{Introduction}

Wireless Underground Sensor Networks (WUSNs) are a promising territory of real-time monitoring and exploration in many underground applications involving intelligent agriculture, pipeline fault diagnosis, subway tunnels, coal mines, earth geology, and earthquake disaster prediction. In this network type, wireless sensor nodes are buried under the ground and exchange sensory information through soil medium $[1,2]$. A primary privilege of using WUSNs is that buried nodes are generally not affecting the functional operations on the ground [3]. In compared with terrestrial WSNs, designing structure in WUSNs is challenging due to the harsh underground environment, the restrictive communication node range, and the difficulty of the sensor energy supplement. Signal propagation among nodes using electromagnetic (EM) wave has been studied in [4-6]. The effect of various factors, such as soil properties in terms of moisture, clay, and sand percentages and system parameters like operating frequency and depth of buried sensor nodes, on the path loss, is also discussed. In [7], authors detail the characteristics of three channels in WUSNs: (1) underground-to-underground channel (UG2UG), (2) underground-to-aboveground channel (UG2AG), and (3) aboveground-to-underground channel (AG2UG).

Usually, direct communications between sensor nodes and aboveground sinks cannot be guaranteed since sensor nodes are possibly buried at deep positions and have limited communication ranges. Consequently, the employment of relay nodes in WUSNs is an encouraging way to achieve high capacity and to extend network coverage. Several works have been proposed regarding the relay employment in WUSNs 
[8-11]. The usage of relay nodes to track water pipes through multilayered underground channel modelling was studied in [8]. This work is aimed at controlling the energy consumed by the buried nodes depending on the optimal positioning of aboveground relay nodes. In [9], the optimal relay node placement that is aimed at prolonging the network's lifetime under limited relay number, load balancing, and network topology constraints is discussed. However, authors in [10] locate virtually changeable relay nodes in two-tier wireless networks in tunnels. Similarly, a hierarchical relay node deployment framework for underground coal mine is suggested in [11]. The sensed data are forwarded through the partitioned regions of the relay nodes which provide efficient coverage for the WUSNs. Although most WSNs research study individually the spectral efficiency (SE), which measures the system data rate in a limited frequency spectrum, and the energy efficiency (EE), which considers the consumed power cost, recent works [12-16] propose to jointly optimize the tradeoff EE-SE between both metrics. However, to the best of our knowledge, this tradeoff optimization has not been yet studied in WUSNs where relay nodes are used. In fact, the throughput optimization in WUSNs, where transmission is based on magnetic induction (MI), is addressed in [17]. Moreover, authors in [18, 19] aim to optimize the throughput in WUSNs where underground sensors harvest RF energy from an aboveground access point. These researches motivate us to study the tradeoff EE-SE optimization in WUSNs in order to enhance the resource allocation and to prolong the lifetime of these networks.

Nowadays, metaheuristic optimization algorithms have been used to solve many complex problems in WSNs by searching for the best solution, among several values, of an optimized objective function known as fitness function. Swarm optimizations and genetic algorithms are the two main categories of metaheuristic algorithms. In the literature, the genetic algorithm (GA) had been used in WUSN optimization to achieve better network lifetime [20,21]. In [20], the wireless sensor nodes of underground mine network are clustered based on GA to obtain a green communication environment. Likewise, the relay node placement in underground room and pillar mines is optimized using GA in [21]. In [22], a single-hop-based routing algorithm for multiple mobile sinks in WSNs is exhibited. The method is composed of two parts: the park position selection based on the Particle Swarm Optimization (PSO) and multipath scheduling for mobile sinks based on the GA. The studies [20-22] target reaching minimized energy and extended network lifetime in WSNs. In $[23,24]$, the Gravitational Search Algorithm (GSA) is used to enhance the energy efficiency of WSNs. In [23], authors calculate the optimal number of clusters and determine the best cluster heads maximizing the efficient energy consumption by applying the novel version of GSA. In [24], authors propose a trust-based energy-aware routing algorithm to select the optimal route from a source to a destination based on energy-aware GSA approach. Another metaheuristic algorithm called Artificial Bee Colony (ABC) has been used for of energy efficient clustering and routing problems in WSNs $[25,26]$. The work [27] suggests the use of Ant Colony Optimization (ACO) to save distance and residual energy of neighbour nodes. Besides, it selects the node with lower energy as next hop, resulting in energy efficient load balancing of the network. A recent metaheuristic algorithm named Salp Swarm Algorithm (SSA) is proposed by [28]. Authors in [28] prove the outperforming of SSA over PSO, GSA, and differential evolution (DE) through experiments on 19 different benchmark functions. Thus, SSA has been used in many fields including engineering issues [29-31], image processing [32], and machine learning [33]. The study [33] integrates SSA with machine learning to achieve automatic image classification. The proposed approach was implemented on three learning algorithms which are Decision Tree, K-nearest Neighbours, and Naïve Bayes. In WSNs, SSA is used to optimize the node localization [34, 35]. Moreover, authors in [36] focused on a security aware and energy efficient multihop routing protocol in WSNs using a modified algorithm named Taylor-based Cat Salp Swarm Algorithm (Taylor C-SSA). In [37], a novel strategy is applied for optimal sensor deployment in WSNs. In this work, an improved version of SSA called weighted SSA (WSSA) based on adding weighted distance position update strategy is proposed. Interestingly, this work proves that even the original SSA algorithm provides energy consumption, coverage, and lifetime optimization compared to many other algorithms like PSO, Whale Optimization Algorithm (WOA), Grey Wolf Optimization (GWO), and Multiverse Optimization (MVO). Therefore, we propose to use the SSA to optimize the resource efficiency in WUSNs employing relay nodes. To the best of our knowledge, our work is the first assessment which studies this topic.

In this paper, we consider a WUSN where underground source nodes transmit sensory information to underground amplify-and-forward (AF) relay nodes through UG2UG communication links. Buried relay nodes retransmit received packets to an aboveground sink node through UG2AG links. To avoid high-power interference signals as discussed in [17], the Time Division Multiple Access (TDMA) scheme is employed. Therefore, nodes use the whole frequency band in their transmissions. We propose to study the EE-SE tradeoff, named resource efficiency (RE), considering the UG2UG and UG2AG link characteristics. The primary objective is to find the optimal source and relay powers required to transmit a new data packet maximizing the total RE of all transmissions that can be performed and considering the initial node battery capacities. Because of the complexity of the considered problem, we use the SSA to optimally estimate the node powers ranging between minimum and maximum allowed values. The complexity of the proposed scheme is studied. The impact of soil composition and system parameters on RE is also well-studied and formulated. Since in some real cases, the composition of underground environment is dynamic and can variate with time [38], we propose to study the case where the soil properties in terms of moisture vary among two successive node transmissions. In addition, an adaptive power allocation, by considering different power constraints among transmissions, is addressed to further improve the RE of the dynamic underground system. The major contributions of our work are listed as follows: 


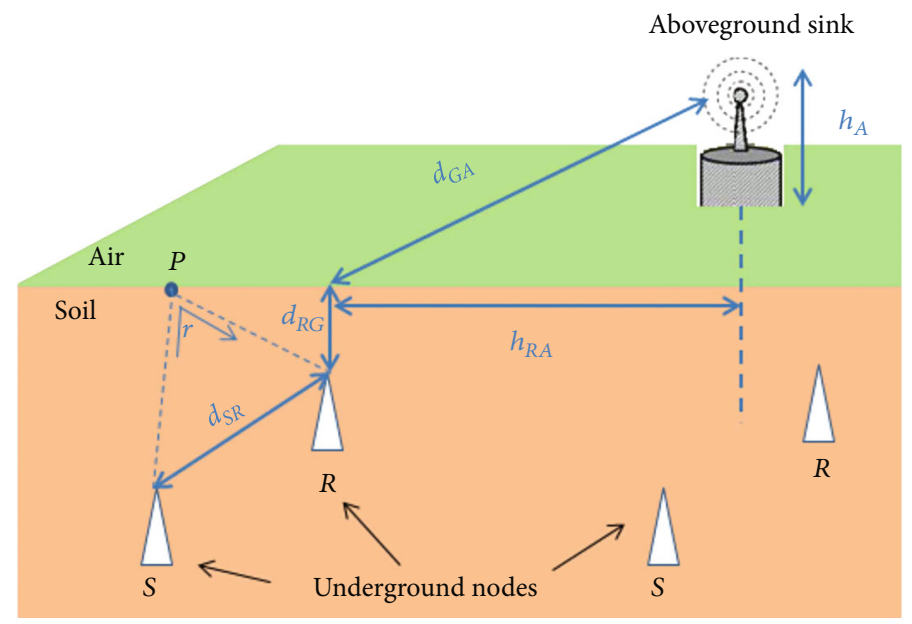

FigURe 1: WUSN topology.

(1) A power allocation scheme maximizing the RE in a WUSN where underground source nodes transmit sensory information to an aboveground sink node through underground relay nodes using UG2UG and UG2AG communication links is proposed

(2) The SSA is used to optimally find the source and relay powers required to transmit a new data packet maximizing the RE and considering minimum and maximum allowed values and the initial node battery capacities

(3) The complexity of the proposed scheme is studied

(4) A significance in RE performance and network's lifetime compared to the conventional WUSN scheme is proved

(5) The proposed scheme is extended to the dynamic underground system where the soil properties in terms of moisture vary among two successive node transmissions

This paper is organized as follows: in Section 2, we present the considered WUSN system model. In Section 3, we detail the channel model for the UG2UG and UG2AG communications. In Section 4, we present the proposed SSAbased power allocation algorithm. In Section 5, we present the simulation results, and in Section 6, we give conclusions.

\section{WUSN System Model}

We consider a WUSN consisting of sensor nodes which collect and transmit sensory information to an aboveground sink node with the help of half-duplex relay nodes. The link between a sensor node and a relay node is an UG2UG link while the communication link connecting a relay node and the aboveground sink node is an UG2AG link as shown in Figure 1. To avoid receiving interference signals, we assume a TDMA scheme. We detail, here, the uplink communication process between a sensor node $S$, a selected AF relay node $R$, and the aboveground sink node $A$. Each node $X \in\{S, R\}$ has a battery with finite power capacity $\mathbf{P}_{X}, X \in\{S, R\}$. Each node $X \in\{S, R\}$ uses some quantity of power $P_{X}^{t}$ when transmitting a new packet $t$ with $t \in[1, T]$, and $T$ is the total number of transmissions that can be performed over the link $(S-R$ - A) under the nodes' initial power capacity constraints $\mathbf{P}_{S}$ and $\mathbf{P}_{R}$. A list of important symbols and notations used in this paper are given in Table 1 .

The communication process requires two steps. In the first step, $S$ transmits a data packet, denoted by $x_{S}^{t}$ to $R$. Hence, the received signal at the relay node $R$ is

$$
\mathrm{y}_{S R}^{t}=\sqrt{P_{S}^{t}} h_{S R}^{t} \mathrm{x}_{S}^{t}+\mathrm{n}_{S R}^{t}
$$

where $h_{S R}^{t}$ is an UG2UG Rayleigh distributed channel between $S$ and $R$ (as explained in [4]) and $n_{S R}^{t}$ is the zeromean complex Additive White Gaussian Noise (AWGN) vector with power spectral density $N_{0}$. In underground transmission, electromagnetic propagation dependents on soil properties and the path loss model are different from the path loss model of the transmission in the air.

The instantaneous signal to noise ratio (SNR) of the transmission between $S$ and $R$ denoted by $\mathrm{SNR}_{S R}^{t}$, is expressed as

$$
\mathrm{SNR}_{S R}^{t}=\frac{P_{S}^{t}\left|h_{S R}^{t}\right|^{2}}{W N_{0}}
$$

where $W$ denotes the channel bandwidth in hertz.

The maximum achievable transmission data rate in bits per second for the transmission between $S$ and $R$, denoted by $R_{S R}^{t}$, is expressed as

$$
R_{S R}^{t}=W \log _{2}\left(1+\mathrm{SNR}_{S R}^{t}\right)
$$


TABLE 1: Symbols and notations.

\begin{tabular}{|c|c|}
\hline Symbol & Definition \\
\hline $\mathbf{P}_{X}$ & Battery power capacity of node $X \in\{S, R\}$ \\
\hline$T$ & Total number of transmissions that can be performed over the link $(S-R-A)$ \\
\hline$P_{X}^{t}$ & Power used to transmit a packet $t \in[1, T]$ \\
\hline$h_{S R}^{t}$ & UG2UG Rayleigh distributed channel between $S$ and $R$ \\
\hline$h_{R A}^{t}$ & UG2AG Rayleigh distributed channel between $R$ and $A$ \\
\hline$n_{X Y}^{t}$ & Zero-mean complex Additive White Gaussian Noise (AWGN) vector with power spectral density $N_{0}$ \\
\hline$W$ & System bandwidth \\
\hline$G^{t}$ & Amplification factor \\
\hline$L_{S R}$ & UG2UG path loss \\
\hline$L_{R A}$ & UG2AG path loss composed by the path losses at the underground and the aboveground portions $L_{R A}^{\mathrm{ug}}$ and $L_{R A}^{\mathrm{ag}}$ \\
\hline$P_{X_{\min }}$ & Minimum power allowed for each transmission \\
\hline$P_{X_{\max }}$ & Maximum power allowed for each transmission \\
\hline$P_{X}^{t *}$ & Optimal power of node $X \in\{S, R\}$ \\
\hline $\mathbf{P}_{X}^{t}$ & Remaining battery capacity of node $X \in\{S, R\}$ at $t \in[1, T]$ \\
\hline $\mathrm{SNR}_{S R A}^{t}$ & Total SNR of the link $(S-R-A)$ \\
\hline$R_{S R A}^{t}$ & Maximum achievable total transmission data rate of the link $(S-R-A)$ \\
\hline$\lambda_{\mathrm{EE}}^{t}$ & Energy efficiency of the link $(S-R-A)$ \\
\hline$\lambda_{\mathrm{SE}}^{t}$ & Spectral efficiency of the link $(S-R-A)$ \\
\hline$\lambda_{\mathrm{RE}}^{t}$ & Resource efficiency of the link $(S-R-A)$ \\
\hline
\end{tabular}

In the second step, $R$ amplifies and retransmits the received signal to $A$. Then, the received signal at $A$ is given by

$$
\mathrm{y}_{R A}^{t}=G^{t} \sqrt{P_{R}^{t}} h_{R A}^{t} y_{S R}^{t}+n_{R A}^{t},
$$

where $h_{R A}^{t}$ is an UG2AG Rayleigh distributed channel between $R$ and $A$ (as explained in [4]), $G^{t}$ is the amplification factor given by $1 / \sqrt{P_{S}^{t}\left|h_{S R}^{t}\right|^{2}+N_{0}}$ and $n_{R A}$ is the zero-mean complex AWGN vector. For soil to air communication, the propagation model is affected by the soil-air interface and the propagation through air.

The instantaneous SNR of the transmission between $R$ and $A$, denoted by $\mathrm{SNR}_{R A}^{t}$, is as follows:

$$
\operatorname{SNR}_{R A}^{t}=\frac{G^{t^{2}} P_{R}^{t} P_{S}^{t}\left|h_{R A}^{t}\right|^{2}\left|h_{S R}^{t}\right|^{2}}{W N_{0}\left(G^{t^{2}} P_{R}^{t}\left|h_{R A}^{t}\right|^{2}+1\right)} .
$$

The maximum achievable transmission data rate in bits per second for the transmission between $R$ and $A$, denoted by $R_{S R}^{t}$, is calculated as

$$
R_{R A}^{t}=W \log _{2}\left(1+\mathrm{SNR}_{R A}^{t}\right) .
$$

Hence, the total SNR of the link $(S-R-A)$, denoted by $\mathrm{SNR}_{S R A}^{t}$, depends on the SNRs of the two hops $\mathrm{SNR}_{S R}^{t}$ and $\mathrm{SNR}_{R A}^{t}$ that is given by

$$
\mathrm{SNR}_{S R A}^{t}=\frac{\mathrm{SNR}_{S R}^{t} \mathrm{SNR}_{R A}^{t}}{1+\mathrm{SNR}_{S R}^{t}+\mathrm{SNR}_{R A}^{t}} .
$$

The maximum achievable total transmission data rate of the link $(S-R-A)$, denoted by $R_{S R A}^{t}$, is expressed as

$$
\begin{aligned}
R_{S R A}^{t} & =W \log _{2}\left(1+\mathrm{SNR}_{S R A}^{t}\right) \\
& =W \log _{2}\left(1+\frac{\mathrm{SNR}_{S R}^{t} \mathrm{SNR}_{R A}^{t}}{1+\mathrm{SNR}_{S R}^{t}+\mathrm{SNR}_{R A}^{t}}\right) .
\end{aligned}
$$

Therefore, the energy efficiency $\lambda_{\mathrm{EE}}^{t}$ of the link $(S-R-A)$ , where energy consumption includes the sensor and the relay node energies, is defined as the total delivered bits per unit energy and is computed as

$$
\lambda_{\mathrm{EE}}^{t}=\frac{R_{S R A}^{t}}{P_{S}^{t}+P_{R}^{t}}=W \frac{\log _{2}\left(1+\mathrm{SNR}_{S R A}^{t}\right)}{P_{S}^{t}+P_{R}^{t}} .
$$


On the other hand, the spectral efficiency $\lambda_{\mathrm{SE}}^{t}$ of the link $(S-R-A)$ is defined as the total delivered bits per unit bandwidth and is given by

$$
\lambda_{\mathrm{SE}}^{t}=\frac{R_{S R A}^{t}}{W}=\log _{2}\left(1+\mathrm{SNR}_{S R A}^{t}\right) .
$$

Finally, the total energy efficiency $\lambda_{\mathrm{EE}}$ and the total spectral efficiency $\lambda_{\mathrm{SE}}$ of the link $(S-R-A)$ over $T$ transmissions are, respectively, given by

$$
\begin{aligned}
& \lambda_{\mathrm{EE}}=\sum_{t=1}^{T} \lambda_{\mathrm{EE}}^{t}, \\
& \lambda_{\mathrm{SE}}=\sum_{t=1}^{T} \lambda_{\mathrm{SE}}^{t} .
\end{aligned}
$$

The mathematical derivations of the UG2UG and UG2AG path losses $L_{S R}$ and $L_{R A}$, of the signal propagation between $S$ and $R$ and between $R$ and $A$, respectively, are detailed in the next section.

\section{Channel Model for the UG2UG and UG2AG Communications}

In WUSN, sensor and relay nodes are both buried into the soil: the relay node is buried close to the ground surface, and the sensor node is deeper as shown in Figure 1. In this scenario, a second path resulting from the wave reflection by ground surface should be considered in addition to the direct path between the two nodes [4]. According to [4], the underground path loss $L_{S R}$ of the channel between the sensor node $S$ and the relay node $R$ is given by

$L_{S R}=6.4+20 \log _{10}\left(d_{S R}\right)+20 \log _{10}(\beta)+8.69 \alpha d_{S R}-10 \log _{10}(V)$,

where $d_{S R}$ is the distance in meter between the sensor node $S$ and the relay node $R$. To simplify the computation, we consider the two-dimensional (2D) plane WUSN deployment which represents the nodes' distances in the plane using Cartesian coordinates defined on $x$ - and $y$-axes. As illustrated in Figure 2, the sink location is the origin of $x$-axis whereas the ground surface is the origin of $y$-axis. The $x_{X}$ is the $x$-axis and $y_{X}$ is the $y$-axis coordinates, which equals the node burial depth $d_{X G}$, of node $X \in\{S, R\}$. Then, $d_{S R}$ is computed as follows

$$
d_{S R}=\sqrt{\left(x_{S}-x_{R}\right)^{2}+\left(y_{S}-y_{R}\right)^{2}} .
$$

According to [4], the factor $V$ is the attenuation factor of the second path given by

$$
\begin{aligned}
V= & +(\Gamma \exp (-\alpha \Delta(r)))^{2} \\
& -2 \Gamma \exp (-\alpha \Delta(r)) \cos \left(\pi-\left(\varnothing-\frac{2 \pi}{\lambda} \Delta(r)\right)\right),
\end{aligned}
$$

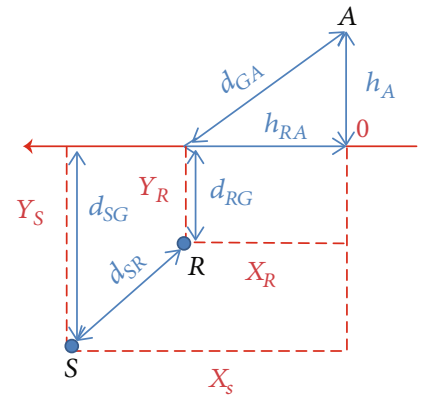

Figure 2: Coordinates of $S$ and $R$.

where $\Gamma$ and $\varnothing$ are the amplitude and phase angle of the reflection coefficient at the reflection point $P, \Delta(r)=r-$ $d_{S R}$ is the difference between the two paths as shown in Figure 1, and $\lambda$ is the wavelength in soil.

The constants $\alpha$, which is the attenuation constant and $\beta$, which is the phase shifting constant, depend on the dielectric properties of soil medium. To calculate these parameters, a model, which describes the electromagnetic propagation considering the soil properties and conditions, is required [39]. The constants $\alpha$ and $\beta$ are, respectively, given by

$$
\begin{aligned}
& \alpha=2 \pi f \sqrt{\frac{\mu_{0} \mu_{r} \epsilon_{0} \epsilon_{1}}{2}\left[\sqrt{1+\left(\frac{\epsilon_{2}}{\epsilon_{1}}\right)^{2}}-1\right]}, \\
& \beta=2 \pi f \sqrt{\frac{\mu_{0} \mu_{r} \epsilon_{0} \epsilon_{1}}{2}\left[\sqrt{1+\left(\frac{\epsilon_{2}}{\epsilon_{1}}\right)^{2}}+1\right]}
\end{aligned}
$$

where $f$ is the operating frequency, $\mu_{0}$ is the free space permeability, $\mu_{r}$ is the soil relative permeability [40], $\epsilon_{0}$ is the permittivity of free space, and $\epsilon_{1}$ and $\epsilon_{2}$ are the real and imaginary parts of the complex soil's dielectric constant given by Peplinski's model [41] as follows:

$$
\begin{aligned}
& \epsilon_{1}=1.15\left[1+\frac{\rho_{b}}{\rho_{s}}\left(\epsilon_{s}^{\alpha_{1}}+m_{v}^{\beta_{1}} \epsilon_{f w 1}^{\alpha_{1}}-m_{v}\right)\right]^{1 / \alpha_{1}}-0.68, \\
& \epsilon_{2}=\left[m_{v}^{\beta_{2}} \epsilon_{f w 2}^{\alpha_{1}}\right]^{1 / \alpha_{1}}
\end{aligned}
$$

where $\rho_{b}$ is the soil bulk density, $\rho_{s}$ is the specific density of the solid soil, $m_{v}$ is the water volume fraction of the mixture, also called the volumetric water content (VWC), and $\alpha_{1}=$ 0.65 is an empirically determined constant. The dielectric constant of soil's solid $\epsilon_{s}$ is

$$
\epsilon_{s}=\left(1.01+0.44 \rho_{s}\right)^{2}-0.062 \text {. }
$$


The parameters $\beta_{1}$ and $\beta_{2}$ in equations (16) and (17) are empirically determined from the sand percentage $S$ and the clay percentage $C$ of the soil and are given by

$$
\begin{aligned}
& \beta_{1}=1.2748-0.519 S-0.152 C, \\
& \beta_{2}=1.3379-0.603 S-0.166 C .
\end{aligned}
$$

Moreover, the real and imaginary parameters $\epsilon_{f w 1}$ and $\epsilon_{f w 2}$ of the relative dielectric constant of free water, mentioned, respectively, in equations (16) and (17), are expressed as

$$
\begin{aligned}
& \epsilon_{f w 1}=\epsilon_{w \infty}+\frac{\epsilon_{w 0}-\epsilon_{w \infty}}{1+\left(2 \pi f \tau_{w}\right)^{2}}, \\
& \epsilon_{f w 2}=\frac{2 \pi f \tau_{w}\left(\epsilon_{w 0}-\epsilon_{w \infty}\right)}{1+\left(2 \pi f \tau_{w}\right)^{2}}+\frac{\sigma_{e f f}}{2 \pi \epsilon_{0} f} \frac{\left(\rho_{s}-\rho_{b}\right)}{\rho_{s} m_{v}},
\end{aligned}
$$

with $\tau_{w}$ is the relaxation time for water, $\epsilon_{w 0}$ is the static dielectric constant for water, and $\epsilon_{w_{\infty}}=4.9$ is the high frequency limit of $\epsilon_{f w 1}$ [42]. Values of $\tau_{w}$ and $\epsilon_{w 0}$ depend on the temperature room. At $20^{\circ} \mathrm{C}, 2 \pi f \tau_{w}=0.58 * 10^{-10}$ and $\epsilon_{w 0}=80.1$. The effective conductivity of $\sigma_{\text {eff }}$ for the $0.3-$ $1.3 \mathrm{GHz}$ band is given by

$$
\sigma_{\text {eff }}=0.0467+0.224 \rho_{b}-0.4111 S+0.6614 C \text {. }
$$

From these equations, it is clear that the path loss model in soil is dependent on the operating frequency $f$, the composition of soil in terms of sand $S$ and clay $C$ percentages, the bulk density $\rho_{b}$, the internode distance $d_{S R}$, and the volumetric water content $m_{v}$.

The communication between the relay node $R$ and the aboveground sink node $A$ consists of two parts, the underground part (soil medium) and the aboveground part (air medium). The refraction loss from underground to the aboveground is absent since the signal propagates perpendicularly from a higher density medium to a lower density medium [7]. Then, based on [7], the path loss $L_{R A}$ of the channel between the relay node $R$ and the aboveground sink node $A$ is given by

$$
L_{R A}=L_{R A}^{\mathrm{ug}}+L_{R A}^{\mathrm{ag}},
$$

where $L_{R A}^{\mathrm{ug}}$ and $L_{R A}^{\mathrm{ag}}$ are the path losses at the underground and the aboveground portions, respectively, and are computed as

$$
\begin{aligned}
& L_{R A}^{\mathrm{ug}}=6.4+20 \log _{10}\left(d_{R G}\right)+20 \log _{10}(\beta)+8.69 \alpha d_{R G}, \\
& L_{R A}^{\mathrm{ag}}=-147.6+10 \eta \log _{10}\left(d_{G A}\right)+20 \log _{10}(f),
\end{aligned}
$$

with $\eta$ is the attenuation coefficient in air with value in the range of 2.8-3.3 according to [43] and $d_{G A}$ is the distance between the relay and the aboveground sink node's antenna given by

$$
d_{G A}=\sqrt{h_{R A}^{2}+h_{A}^{2}}
$$

where $h_{R A}$ is the horizontal distance between the relay node $R$ and the aboveground sink node $A$ and $h_{A}$ is the height of the aboveground sink node $A$.

When TDMA is used in WUSNs, nodes transmit packets using the whole frequency band in different time slots. Then, the operating frequency $f$ is set to the system bandwidth.

\section{Optimization Problem and Proposed Power Allocation Algorithm}

In this section, we review first the algorithm SSA employed in the EE-SE tradeoff optimization. Then, the considered problem is formulated assuming a static underground environment. At the end of this section, we propose to adapt the proposed algorithm to the case where the soil property in terms of VWC varies among two successive node transmissions.

4.1. Salp Swarm Algorithm (SSA). One of the latest metaheuristic swarm algorithms, that successfully have been employed to optimize different problems in several research areas, is the SSA [44]. This algorithm was firstly introduced by Seyedali Mirjalili in 2017 which mimics the floating of salps that navigate in aquarium environments such as oceans and seas [28]. In deep oceans, salps compose a swarm which is called salp chain. The algorithm divides the populations of salps (i.e., search agents) into two groups: the leader and the followers. The leader salp is at the beginning of the chain and guides the salp population while the rest are followers since they follow the leader.

SSA is simple and like other swarm-based intelligent algorithms. The position of $N$ salps is randomly initialized in an $n$ dimensional search space where $n$ is the number of variables of the considered problem. Additionally, the positions of salps are stored in a two-dimensional matrix called $x$. A food source called $\mathrm{fd}$ in the search space is defined as the swarm's target. Then, the fitness of each salp is determined to select the leader with the best fitness. The position of the leader is updated using the following equation.

$$
x_{j}^{1}= \begin{cases}\mathrm{fd}_{j}+c_{1}\left(\left(\mathrm{ub}_{j}-\mathrm{lb}_{j}\right) c_{2}+\mathrm{lb}_{j}\right) & c_{3} \geq 0, \\ \mathrm{fd}_{j}-c_{1}\left(\left(\mathrm{ub}_{j}-\mathrm{lb}_{j}\right) c_{2}+\mathrm{lb}_{j}\right) & c_{3}<0,\end{cases}
$$

where the $x_{j}^{1}$ is the position of the leader in the $j^{\text {th }}$ dimension, $\mathrm{fd}_{j}$ is the food source in the $j^{\text {th }}$ dimension, $\mathrm{lb}_{j}$ and $\mathrm{ub}_{j}$ are the lower bound and the upper bound of the $j^{\text {th }}$ dimension, respectively, $c_{2}$ and $c_{3}$ are random numbers between $[0,1]$, and $c_{1}$ is defined as follows:

$$
c_{1}=2 e^{-(4 l / L)^{2}},
$$


with $l$ is the current iteration and $L$ is the maximum number of iterations. The position of the $i^{\text {th }}$ follower is updated using the equation given by

$$
x_{j}^{i}=\frac{1}{2}\left(x_{j}^{i}+x_{j}^{i-1}\right) \quad i \geq 2,
$$

where $x_{j}^{i}$ is the position of the $i^{\text {th }}$ follower in the $j^{\text {th }}$ dimension.

Using equations (25) and (27), the positions of the salp chain population are updated until the suitable solution is obtained or a stopping criterion is met.

4.2. Optimization Problem and Proposed Algorithm. As the conventional design using $\lambda_{\mathrm{EE}}^{t}$ or $\lambda_{\mathrm{SE}}^{t}$ is limited in respect of the inefficient use of resources, we are interested to determine the power allocation vector $\mathbf{P}=\left[P_{S}^{t}, P_{R}^{t}\right]$ at each $t$ transmission performed over the link $(S-R-A)$ that jointly maximizes the energy efficiency $\lambda_{\mathrm{EE}}^{t}$ and the spectral efficiency $\lambda_{\mathrm{SE}}^{t}$ for all transmissions $t \in[1, T]$. Since nodes are equipped with batteries with finite capacities, the optimization of the power $P_{X}^{t}$ of each node $X \in\{S, R\}$, used at the $t^{\text {th }}$ transmission, should consider the initial battery capacity $\mathbf{P}_{X}$, the spent powers in previous transmissions $\left[P_{X}^{1}, \cdots, P_{X}^{t-1}\right]$, and the power limitation range $\left[P_{X_{\min }}, P_{X_{\max }}\right]$ allowed for each transmission. The proposed multiobjective optimization problem is as follows:

$$
\begin{aligned}
P_{S}^{t *}, P_{R}^{t *}= & \max \sum_{t=1}^{T}\left(\lambda_{\mathrm{EE}}^{t}\left(P_{S}^{t}, P_{R}^{t}\right), \lambda_{\mathrm{SE}}^{t}\left(P_{S}^{t}, P_{R}^{t}\right)\right) \\
& \text { Subject to } \sum_{t=1}^{T} P_{X}^{t} \leq \mathbf{P}_{X} .
\end{aligned}
$$

where $P_{X}^{t} \in\left[P_{X_{\min }}, P_{X_{\max }}\right]$ for $X \in[S, R]$.

Since optimizing simultaneously two functions which are competing is challenging, we propose to optimize the unique weighted objective function, called the resource efficiency $\lambda_{\mathrm{RE}}^{t}$, exploiting the tradeoff between $\lambda_{\mathrm{EE}}^{t}$ and $\lambda_{\mathrm{SE}}^{t}$ as discussed in the work [12]. The resource efficiency $\lambda_{\mathrm{EE}}^{t}$ of the link ( $S$ $-R-A)$ at the $t^{\text {th }}$ transmission, for $t \in[1, T]$, is given by

$$
\lambda_{\mathrm{RE}}^{t}=\lambda_{\mathrm{EE}}^{t}+\omega \lambda_{\mathrm{SE}}^{t}
$$

where the factor $\omega$ is computed as

$$
\omega=\bar{\omega} \frac{W}{P_{\text {tot }}},
$$

with $\bar{\omega}$ is a constant, $W$ is the available bandwidth, and $P_{\text {tot }}$ is the total power budget allocated to the link $(S-R-A)$ at each transmission. Then, $P_{\text {tot }}=P_{S_{\max }}+P_{R_{\max }}$. The weighted factor $\omega$ is introduced to ensure the balancing between $\lambda_{\mathrm{EE}}^{t}$ and $\lambda_{\mathrm{SE}}^{t}$ because $\lambda_{\mathrm{SE}}^{t}$ is practically smaller than $\lambda_{\mathrm{EE}}^{t}$. Moreover, $\omega$ corrects the inconsistence of adding two metrics with different units since the unit of $\lambda_{\mathrm{EE}}^{t}$ is bits/joule while the unit of $\lambda_{\mathrm{SE}}^{t}$ is bits/s/Hz. Then, the unit of $\lambda_{\mathrm{RE}}^{t}$ is similar to $\lambda_{\mathrm{EE}}^{t}$ which is still bits/joule. Besides, optimizing $\lambda_{\mathrm{RE}}^{t}$ tends to optimize $\lambda_{\mathrm{EE}}^{t}$ if $\bar{\omega}=0$ and to optimize $\lambda_{\mathrm{SE}}^{t}$ if $\bar{\omega}=\infty$. Generally, the choice of the value of this constant is based on the designer objective and the available resources in terms of bandwidth and power [12]. We propose to evaluate this constant for the considered WUSN in Section 5.

Consequently, the constrained multiobjective optimization problem in equation (28) is transformed into a singleobjective problem as follows: where

$$
\begin{gathered}
P_{S}^{t *}, P_{R}^{t *}=\max \sum_{t=1}^{T} \lambda_{\mathrm{RE}}^{t}\left(P_{S}^{t}, P_{R}^{t}\right) \\
\text { Subject to } \sum_{t=1}^{T} P_{X}^{t} \leq \mathbf{P}_{X}, \\
P_{X}^{t} \in\left[P_{X_{\min }}, P_{X_{\max }}\right] \text { for } X \in[S, R] .
\end{gathered}
$$

The considered problem in equation (31) is NP-hard since the number of variables to optimize equals two $\left(P_{S}^{t}\right.$ and $P_{R}^{t}$ ) and the presence of variable products in equations (5) and (7) caused by the AF relay transmission. Therefore, the metaheuristic algorithm SSA is used to compute the optimal solution of the problem. The followed procedure is presented below.

Step 1. Each node $X \in\{S, R\}$ has a finite battery capacity $\mathbf{P}_{X}$. We define $\mathbf{P}_{X}^{t}$ as the remaining battery capacity of node $X \in[S, R]$ at the $t^{\text {th }}$ transmission. Before transmitting, $\mathbf{P}_{X}^{0}=\mathbf{P}_{X}$.

Step 2. The number of variables is $n$ is set to $2: P_{S}^{t}$ and $P_{R}^{t}$. The upper bounds $\mathrm{ub}_{1}$ (of the variable $P_{S}^{t}$ ) and $\mathrm{ub}_{2}$ (of the variable $P_{R}^{t}$ ) are set, respectively, to $P_{S_{\max }}$ and $P_{R_{\max }}$. The lower bounds $\mathrm{lb}_{1}$ (of the variable $P_{S}^{t}$ ) and $\mathrm{lb}_{2}$ (of the variable $P_{R}^{t}$ ) are set, respectively, to $P_{S_{\min }}$ and $P_{R_{\min }}$. The number of search agent (salps) $N$ is fixed.

Step 3. The objective (fitness) function is formulated as

$$
F\left(P_{S}^{t}, P_{R}^{t}\right)=\max \left(\lambda_{\mathrm{RE}}^{t}\left(P_{S}^{t}, P_{R}^{t}\right)\right) .
$$

Step 4. Optimal node powers $P_{S}^{t *}$ and $P_{R}^{t *}$ are determined.

Step 5. The remaining battery capacity is decremented as follows:

$$
\mathbf{P}_{X}^{t+1}=\mathbf{P}_{X}^{t}-P_{X}^{t^{*}}, \quad X \in[S, R] .
$$

Step 6. Repeat the steps 3-6 until $P_{S}^{t} \leq P_{S_{\min }}$ or $P_{R}^{t} \leq P_{R_{\min }}$.

The SSA can be implemented at the sink node $A$ which is assumed to have perfect channel knowledge. After that, computed optimal powers are sent to sensor and relay nodes prior to their transmissions. 
4.3. Computational Complexity Analysis. We propose, in this section, to evaluate the complexity of the proposed power allocation scheme. First, it requires a constant running time to initialize the system parameters which can be expressed as $O(1)$ time. At each $t \in[1, T]$ transmission, the SSA algorithm is computed. The computational complexity of SSA is generally written in [28] as follows $O(L(n N+\operatorname{Cof} N))$, where $L$ is the total iterations, $n$ is the number of variables, Cof is the cost of the objective function, and $N$ is the salp population. In our case, the number of variables $n$ is set to 2; then, the running time of the SSA algorithm is $O(L(2 N$ $+\operatorname{Cof} N)$ ). The total complexity of SSA computation over $T$ transmissions is $O(T L(2 N+\operatorname{Cof} N))$. The computation of the remaining battery capacity is done at each transmission for both relay and source nodes in equation (33). Then, the required time for this operation is $O(2 T)$. Hence, the total running time of the proposed algorithm is $O(1)+O(T L(2 N$ $+\operatorname{Cof} N))+O(2 T)$.

4.4. Case of Dynamic Underground Environment. In the previous section, we assumed that the underground environment is unchanged with time. However, in some real cases, the water content in the soil can variate due to the soil irrigation or to the weather change during different seasons [38]. We propose, here, to study the case where the soil composition in terms of VWC varies among two successive node transmissions. In addition, an adaptive power allocation based on assigning different power constraints to different node transmissions is suggested. Therefore, the optimization problem is as follows:

$$
\begin{aligned}
P_{S}^{t^{*}}, P_{R}^{t^{*}}= & \max \sum_{t=1}^{T} \lambda_{\mathrm{RE}}^{t}\left(P_{S}^{t}, P_{R}^{t}\right) \\
& \text { Subject to } \sum_{t=1}^{T} P_{X}^{t} \leq \mathbf{P}_{X} .
\end{aligned}
$$

where $P_{X}^{t} \in\left[P_{X_{\min }}, P_{X_{\max }}^{t}\right]$ for $X \in[S, R]$, and

$$
P_{X_{\max }}^{t}=\delta^{t} \mathbf{P}_{X} \text { for } X \in[S, R]
$$

where $\delta^{t}$ is the power fraction assigned to the $t^{\text {th }}$ transmission for $t \in[1, T]$ and $\sum_{t=1}^{T} \delta^{t}=1$. Here, the same procedure, described in Section 4.2, is followed considering a given maximum power $P_{X_{\max }}^{t}$ for each $t$ transmission.

\section{Numerical Results and Discussions}

In this section, we present the simulation results illustrating the performance of the proposed power allocation algorithm. Simulations are performed using MATLAB-R2015a simulator on a Windows 7 OS with 2 GB RAM memory. Table 2 summarizes the list of parameters used in the path loss computation and their values. All simulation results are calculated by averaging over 1000 channel realizations. The $x$ -axis coordinates $x_{X}$ of node $X \in[S, R]$ is set to $0.2 \mathrm{~m}$. The depth of node $X \in[S, R]$ equals its $y$-axis coordinate: $d_{\mathrm{XG}}=$
TABLE 2: Values of path loss parameters.

\begin{tabular}{lc}
\hline Parameters & Values \\
\hline$S$ & $5 \%$ \\
$C$ & $15 \%$ \\
$\mu_{0}$ & $4 \pi 10^{-7} \mathrm{~N} / \mathrm{A}^{2}$ \\
$\mu_{r}$ & 1 \\
$\epsilon_{0}$ & $8.85 * 10^{-12} \mathrm{~F} / \mathrm{m}$ \\
$\rho_{b}$ & $1.5 \mathrm{~g} / \mathrm{cm}^{3}$ \\
$\rho_{s}$ & $2.66 \mathrm{~g} / \mathrm{cm}^{3}$ \\
$m_{v}$ & $5 \%-25 \%$ \\
$\tau_{w}$ & $(1 / 2 \pi f) 0.58 * 10^{-10} \mathrm{~s}$ \\
$\epsilon_{w 0}$ & 80.1 \\
$\epsilon_{w \infty}$ & 4.9 \\
$\eta$ & 3 \\
$W$ & $0.3-1.3 \mathrm{GHz}$ \\
$N_{0}$ & $-130 \mathrm{dBm} / \mathrm{Hz}$ \\
$\Gamma$ & 5 \\
$\varnothing$ & $60^{\circ}$ \\
\hline
\end{tabular}

$y_{X}$. The height $h_{A}$ of $A$ is set to $0.7 \mathrm{~m}$. We assume that the batteries of source and relay nodes have equal power capacities $\mathbf{P}_{S}=\mathbf{P}_{R}=\mathbf{P}$. For Figures 3-9 and Table 3, we assume that underground medium conditions are unchanged with time. We also consider that the physical limitations of each transmission power are $P_{S_{\min }}=P_{R_{\min }}=P_{\min }=5 \mathrm{mw}$ and $P_{S_{\max }}=P_{R_{\max }}=P_{\max }$.

Figure 3 illustrates the convergence of the resource efficiency $\lambda_{\mathrm{RE}}$ as a function of the number of iterations for different numbers of search agents (salps) $N$ used by the SSA algorithm in the optimal node power computation. We consider that $S$ and $R$ are buried at depths $d_{S G}=0.6 \mathrm{~m}$ and $d_{R G}$ $=0.1 \mathrm{~m}$, respectively. The node battery capacity $\mathbf{P}$ is set to $3 \mathrm{~V}$, and the maximum allowed power for each transmission $P_{\max }$ is set to $50 \mathrm{mw}$. The VWC $m_{v}$ is set to $5 \%$. The system bandwidth $W$ is set to $300 \mathrm{MHz}$. As it is clearly shown, increasing the number of search agents enhances the computation accuracy of the maximum of $\lambda_{\mathrm{RE}}$. Also, the convergence of the SSA algorithm is fast as less than five iterations are required to reach the optimal values of $\lambda_{\mathrm{RE}}$ for all values of $N$. Therefore, the algorithm requires small memory storage, and it can be easily implemented with low cost of computational complexity. We consider that $N=20$ in the rest of simulation results.

Table 3 shows the resource efficiency $\lambda_{\mathrm{RE}}$ statistical results, using a set of performance measures, of the proposed SSA-based optimization scheme compared to the conventional WUSN scheme with fixed node powers. We consider $\mathbf{P}=3 \mathrm{~V}, P_{\max }=50 \mathrm{mw}, W=300 \mathrm{MHz}, d_{S G}=0.6 \mathrm{~m}, d_{R G}=$ $0.1 \mathrm{~m}$, and $m_{v}=5 \%$. For the conventional WUSN scheme with fixed powers, we adopt equal node power allocation set to the maximum allowed powers, $P_{X}^{t}=P_{\max }$ for $X \in[S, R]$ and $t \in[1, T]$. The performance measures are the maximum 


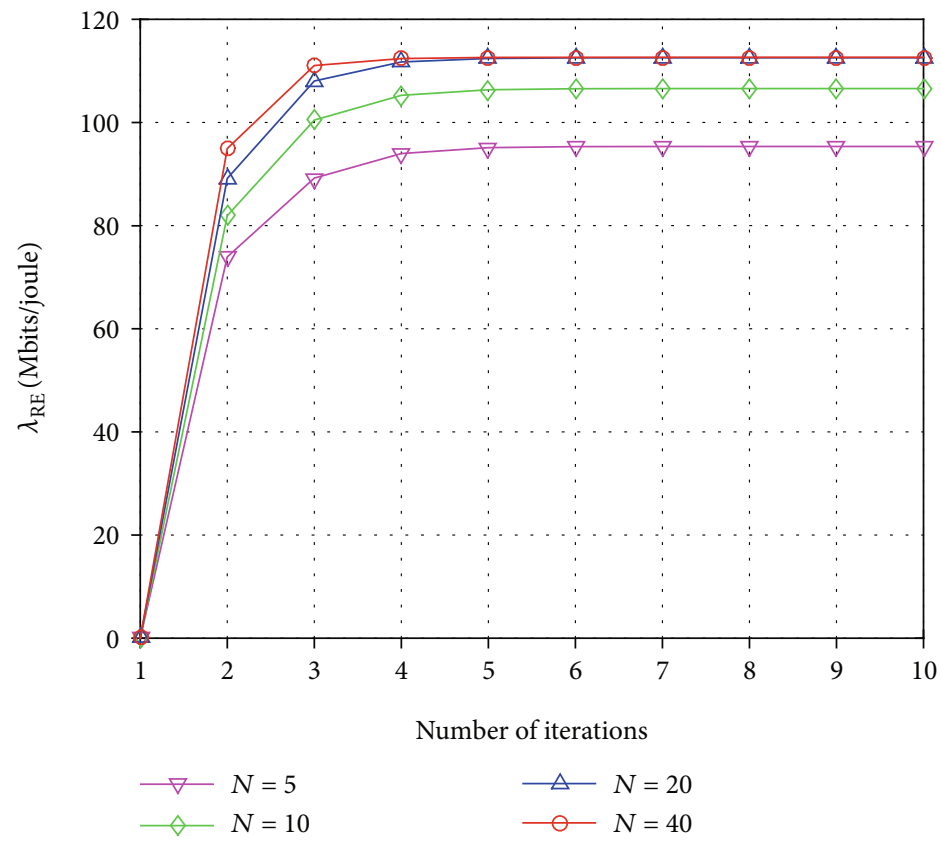

FIGURE 3: Convergence behavior of $\lambda_{\mathrm{RE}}$ versus the number of iterations for different numbers of search agents $N$ with $d_{S G}=0.6 \mathrm{~m}, d_{R G}=0.1 \mathrm{~m}$, $\mathbf{P}=3 \mathrm{~V}, P_{\max }=50 \mathrm{mw}, W=300 \mathrm{MHz}$, and $m_{v}=5 \%$.

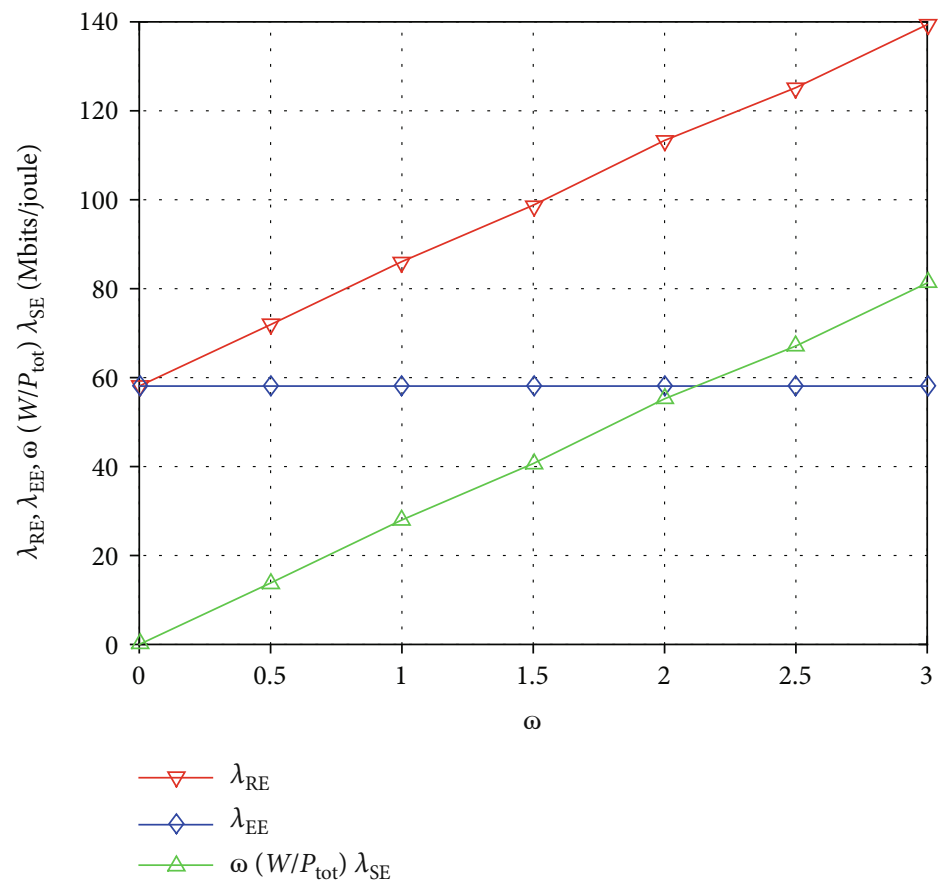

Figure 4: Impact of weighted factor $\bar{\omega}$ on $\lambda_{\mathrm{RE}}$ with $d_{S G}=0.6 \mathrm{~m}, d_{R G}=0.1 \mathrm{~m}, \mathbf{P}=3 \mathrm{~V}, P_{\max }=50 \mathrm{mw}, W=300 \mathrm{MHz}$, and $m_{v}=5 \%$.

( $\max$ ), the minimum ( $\min )$, the standard deviation (Std), and the average (mean) with confidence value. The confidence value is computed using the confidence level 95\%. Compared to the conventional WUSN scheme, the proposed SSA-based optimization scheme achieves better performance for all evaluated performance measures.

Figure 4 shows the variation of the resource efficiency $\lambda_{\mathrm{RE}}$ , the energy efficiency $\lambda_{\mathrm{EE}}$, and the weighted spectral effi- ciency $\bar{\omega}\left(W / P_{\text {tot }}\right) \lambda_{\mathrm{SE}}=\omega \lambda_{\mathrm{SE}}$ versus the weighted factor $\bar{\omega}$. We consider $d_{S G}=0.6 \mathrm{~m}, d_{R G}=0.1 \mathrm{~m}, \mathbf{P}=3 \mathrm{~V}, P_{\max }=50$ $\mathrm{mw}, W=300 \mathrm{MHz}$, and $m_{v}=5 \%$. The $\lambda_{\mathrm{EE}}$ is constant since it does not depend on $\bar{\omega}$. The weighted spectral efficiency $\omega$ $\lambda_{\mathrm{SE}}$ increases with the increasing of $\bar{\omega}$. As expected, when $\bar{\omega}$ is small, optimizing $\lambda_{\mathrm{RE}}$ leads to simply optimizing $\lambda_{\mathrm{EE}}$ and when $\bar{\omega}$ is larger, more weight is added to $\lambda_{\mathrm{SE}}$ and balancing between $\lambda_{\mathrm{EE}}$ and $\lambda_{\mathrm{SE}}$ is more insured. Clearly, when $\bar{\omega}=2.1$, 


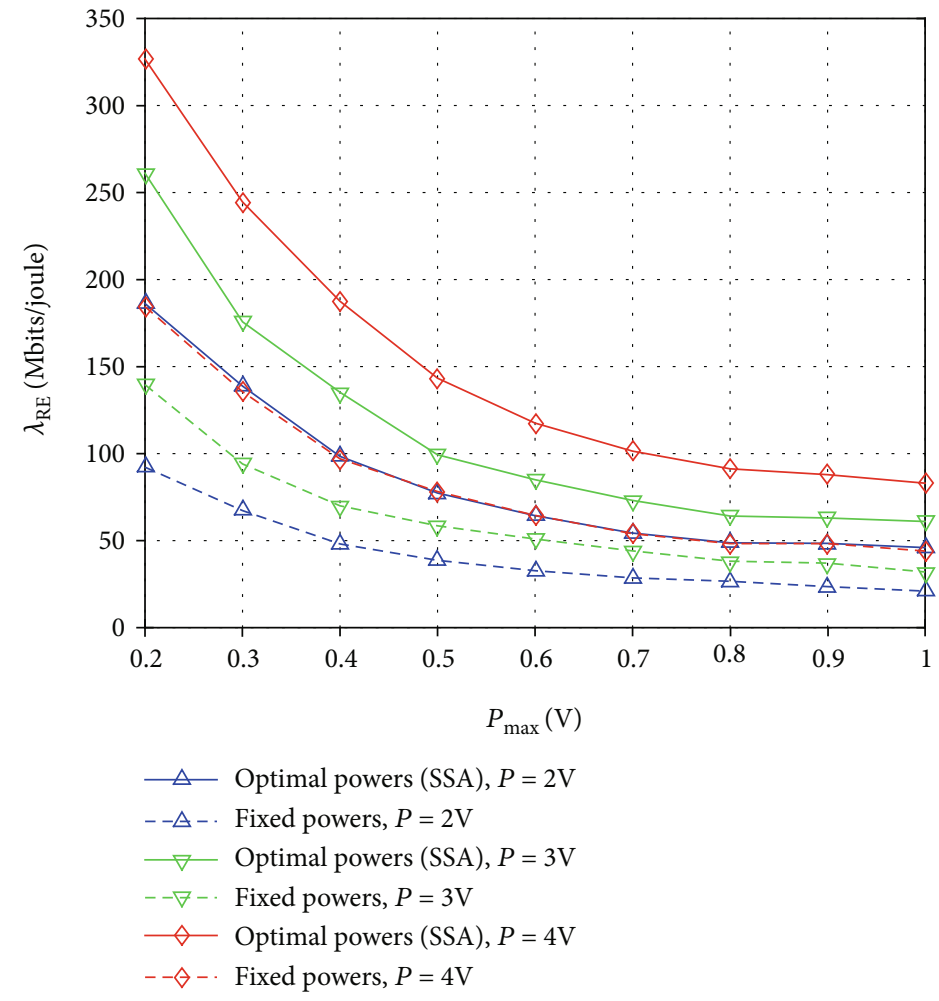

FIGURE 5: Resource efficiency of the proposed power allocation scheme compared to conventional WUSN scheme with fixed powers versus the maximum allowed power $P_{\max }$ for different battery capacities $\mathbf{P} \in\{2,3,4\}$ with $d_{S G}=0.6 \mathrm{~m}, d_{R G}=0.1 \mathrm{~m}, W=300 \mathrm{MHz}$, and $m_{v}=5 \%$.

$\lambda_{\mathrm{RE}}$ results from the best balancing between $\lambda_{\mathrm{EE}}$ and $\lambda_{\mathrm{SE}}$. We notice that the weighted factor $\bar{\omega}$ is here greater than the weighted factor in [12]. Indeed, the nature of the underground medium in WUSNs causes lower $\lambda_{\mathrm{SE}}$ than that in WSNs considered in the work [12]. Hence, a higher weighted factor is needed in the case of WUSNs. As a result, we set $\bar{\omega}$ to 2.1 in the rest of simulation results.

Figure 5 exhibits the resource efficiency $\lambda_{\mathrm{RE}}$ performance of the proposed node power optimization scheme compared to the conventional WUSN scheme with fixed node powers versus the maximum power allowed for each transmission $P_{\max }$ for different power capacities $\mathbf{P} \in\{2,3,4\}$. We consider $d_{S G}=0.6 \mathrm{~m}, d_{R G}=0.1 \mathrm{~m}, W=300 \mathrm{MHz}$, and $m_{v}=5 \%$. Compared to the conventional WUSN scheme, the proposed scheme with optimal node powers provides a better resource efficiency for the same total power cost. Indeed, the proposed power allocation scheme improves efficiently the total number of delivered bits with considering the optimal node power distribution according to the power physical limitations. The resource efficiency $\lambda_{\mathrm{RE}}$ increases when the power capacity $\mathbf{P}$ of node batteries increases. Moreover, the resource efficiency $\lambda_{\mathrm{RE}}$ decreases when $P_{\max }$ increases. In fact, increasing the maximum power $P_{\max }$ harms the energy efficiency $\lambda_{\mathrm{EE}}$ and the weighted spectral efficiency $\bar{\omega}\left(W / P_{\text {tot }}\right) \lambda_{\mathrm{SE}}$ as well. Consequently, for a given resource efficiency requirement, designer can adjust the maximum power allowed for each transmission depending on the available resource in terms of battery capacities.

Figure 6 shows the resource efficiency $\lambda_{\mathrm{RE}}$ performance of the proposed node power optimization scheme compared to the conventional WUSN scheme with fixed node powers versus the system bandwidth $W$ for different battery capacities $\mathbf{P} \in\{2,3,4\}$. We consider $d_{S G}=0.6 \mathrm{~m}, d_{R G}=0.1 \mathrm{~m}$, $P_{\max }=50 \mathrm{mw}$, and $m_{v}=5 \%$. Clearly, the proposed scheme enhances the $\lambda_{\mathrm{RE}}$ performance compared to the conventional WUSN scheme for the same used system bandwidth $W$. According to Shannon's formula, traditional design focuses on expanding the bandwidth and reducing the transmit power to achieve a target data rate requirement. Hence, as the bandwidth increases, the $\lambda_{\mathrm{EE}}$ increases whereas the $\lambda_{\mathrm{SE}}$ decreases. As shown in the figure, increasing the system bandwidth harms the EE-SE tradeoff performance of the considered WUSN. Indeed, the increase of the operating frequency increases the UG2UG and UG2AG path losses and mitigates the channels as demonstrated in [4]. Hence, the use of lower bandwidths is better for the considered WUSN where relay nodes are deployed.

Figure 7 illustrates the impact of the proposed power allocation scheme on the number of transmissions $T$ that can be performed through the link $S-R-A$ for different sensor depths $d_{S G} \in[0.3 \mathrm{~m}, 0.6 \mathrm{~m}]$ and different relay depths $d_{R G} \in\{0.05 \mathrm{~m}, 0.1 \mathrm{~m}, 0.2 \mathrm{~m}\}$. We consider $\mathbf{P}=3 \mathrm{~V}, P_{\max }=$ $50 \mathrm{mw}, W=300 \mathrm{MHz}$, and $m_{v}=5 \%$. Clearly, the number of transmissions $T$ is enhanced using the proposed node power optimization. It is more improved when $S$ is closer to $R$ and $R$ is closer to the ground surface. Thus, the proposed power allocation scheme efficiently extends the node battery lifetime and additionally the network lifetime.

Figure 8 presents the resource efficiency $\lambda_{\mathrm{RE}}$ performance of the proposed node power optimization scheme and the 


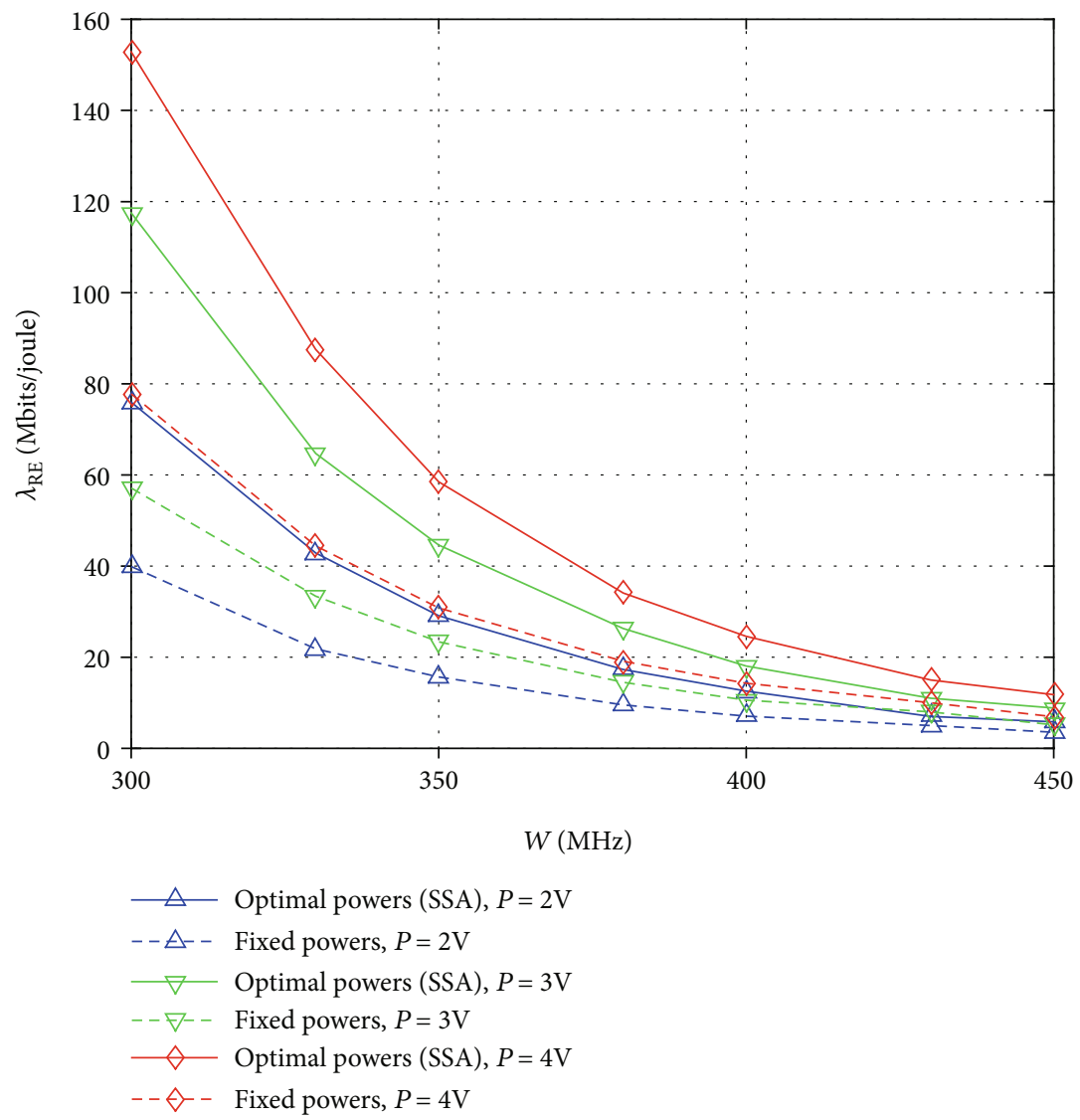

FIGURE 6: Resource efficiency of the proposed power allocation scheme compared to conventional WUSN scheme with fixed powers versus the system bandwidth $W$ for different battery capacities $\mathbf{P} \in\{2,3,4\}$ with $d_{S G}=0.6 \mathrm{~m}, d_{R G}=0.1 \mathrm{~m}, W=300 \mathrm{MHz}$, and $m_{v}=5 \%$.

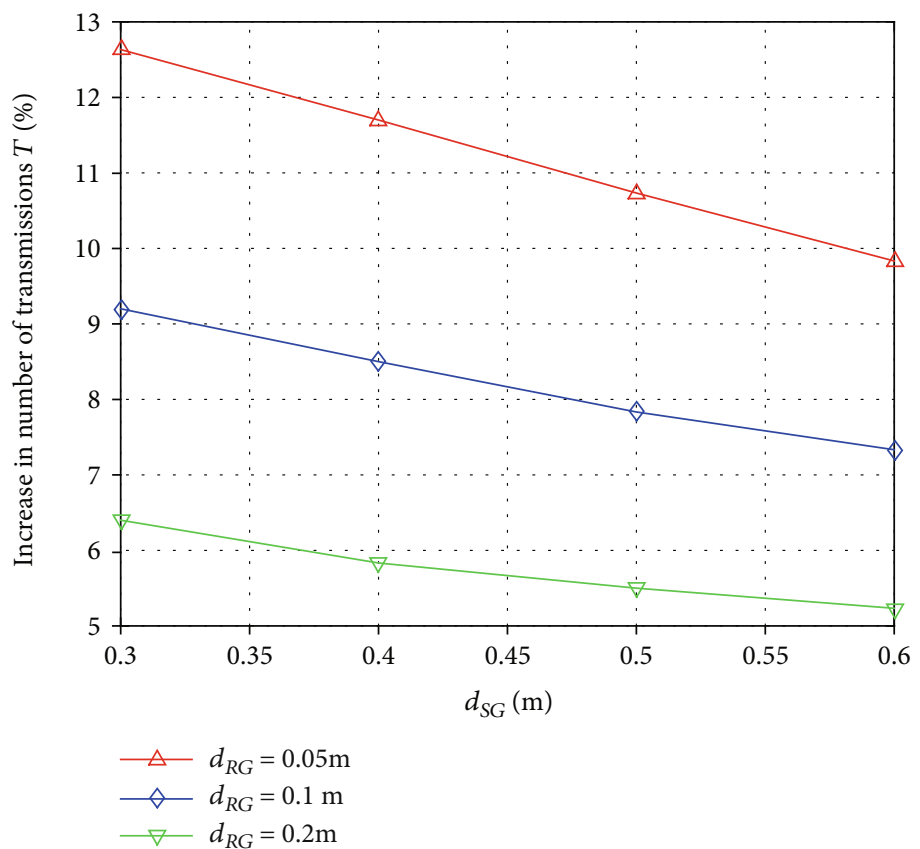

FIGURE 7: Increase in number of transmissions $T$ of the proposed power allocation scheme compared to the conventional WUSN scheme with fixed powers for different node burial depths $d_{S G}$ and $d_{R G}$ configurations with $\mathbf{P}=3 \mathrm{~V}, P_{\max }=50 \mathrm{mw}, W=300 \mathrm{MHz}$, and $m_{v}=5 \%$. 


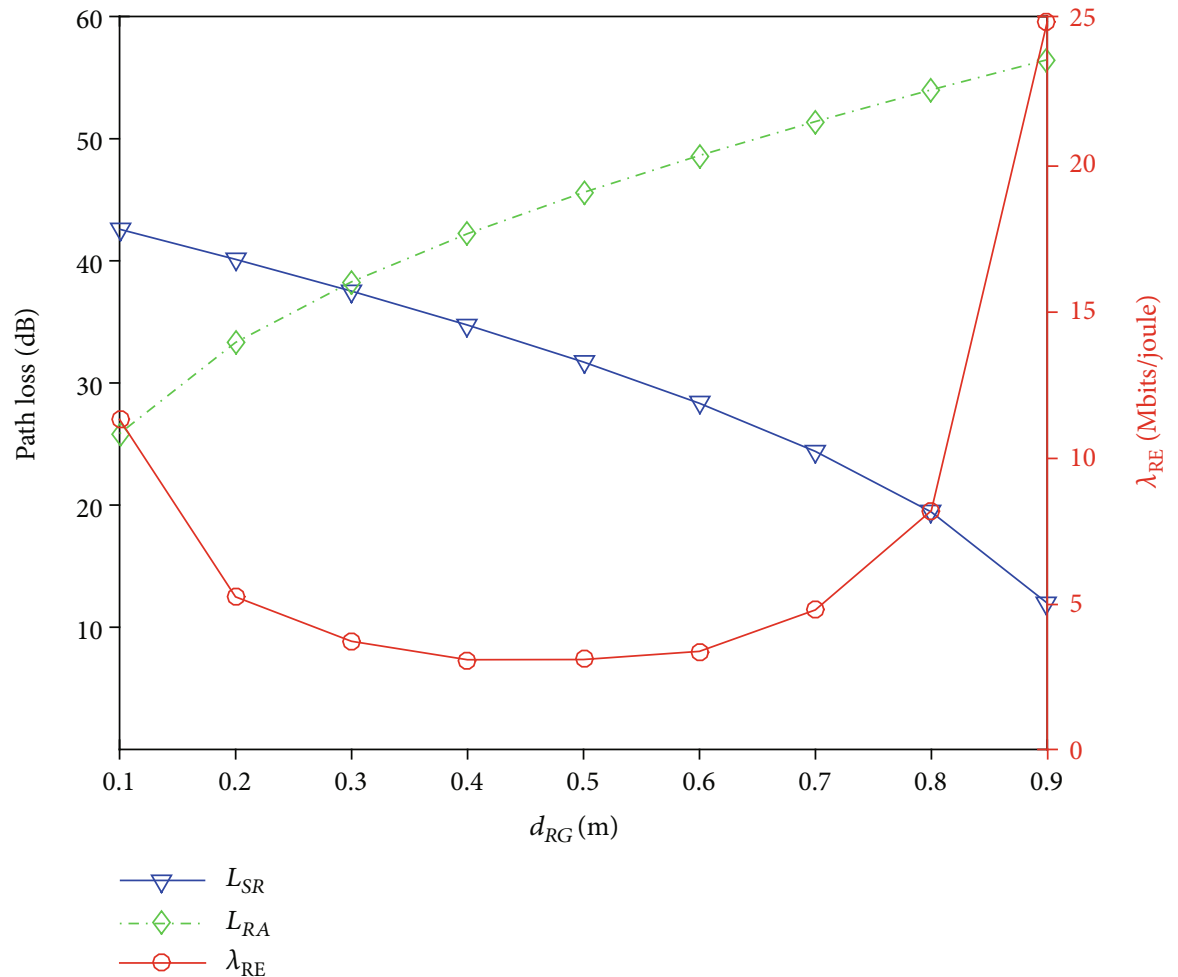

FIGURE 8: Resource efficiency of the proposed power allocation scheme, the UG2UG path loss $L_{S R}$ and the UG2AG path loss $L_{R A}$ versus the relay depth $d_{R G}$ with $d_{S G}=1 \mathrm{~m}, \mathbf{P}=3 \mathrm{~V}, P_{\max }=50 \mathrm{mw}, W=300 \mathrm{MHz}$, and $m_{v}=5 \%$.

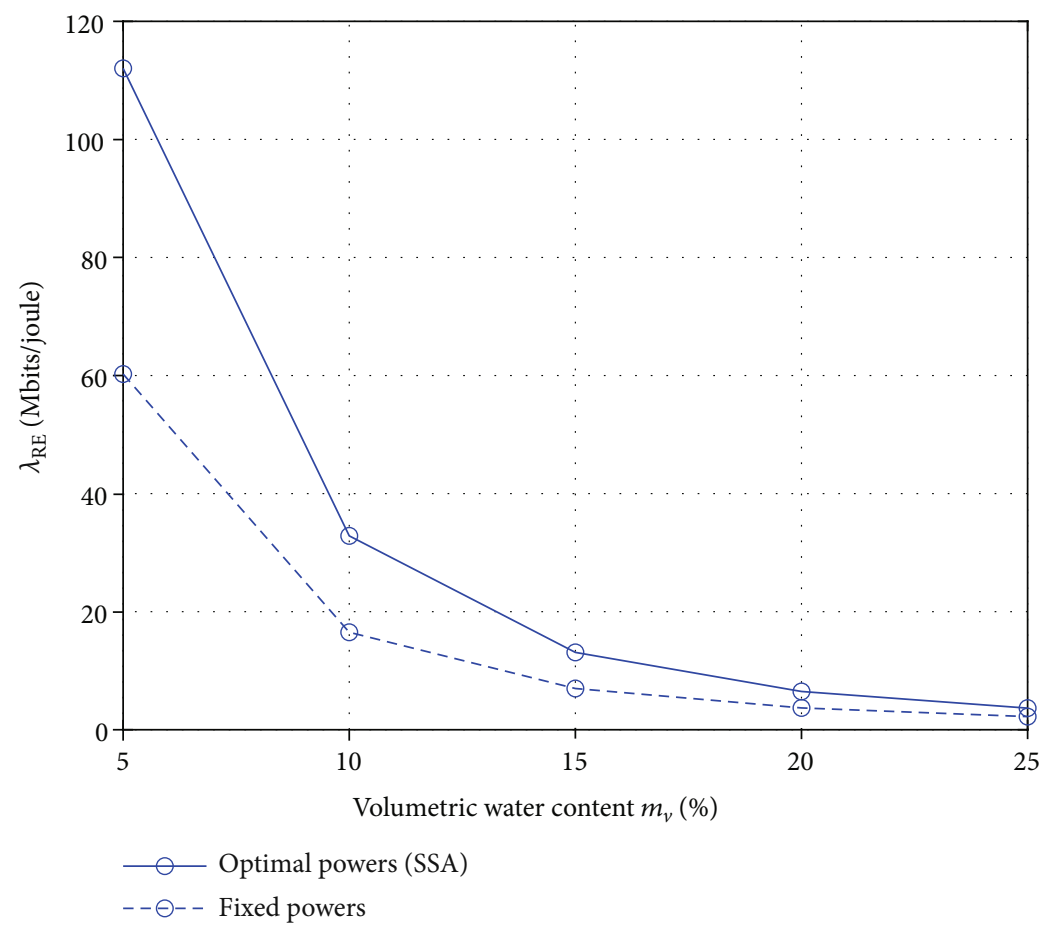

FIGURE 9: Resource efficiency of the proposed power allocation scheme compared to conventional WUSN scheme with fixed powers versus the volumetric water content (VWC) $m_{v}$ with $d_{S G}=0.6 \mathrm{~m}, d_{R G}=0.1 \mathrm{~m}, \mathbf{P}=3 \mathrm{~V}, P_{\max }=50 \mathrm{mw}$, and $W=300 \mathrm{MHz}$.

variation of the UG2UG path loss $L_{S R}$ and the UG2AG path loss $L_{R A}$ for different relay depths $d_{R G} \in[0.1 \mathrm{~m}, 0.9 \mathrm{~m}]$. We assume that the sensor $S$ is buried at fixed depth $y_{S}=d_{S G}=$
$1 \mathrm{~m}$. We consider $\mathbf{P}=3 \mathrm{~V}, P_{\max }=50 \mathrm{mw}, W=300 \mathrm{MHz}$, and $m_{v}=5 \%$. The increase of the burial relay depth $d_{R G}$ means that $R$ is closer to $S$ and further from the ground 
TABLE 3: Statistical results.

\begin{tabular}{lcccc}
\hline Scheme & Max & Min & Std & Mean \pm confidence \\
\hline$\lambda_{\mathrm{RE}}$ using SSA-based optimization (Mbits/joule) & 778.6842 & 4.6895 & 87.1813 & $114.9157 \pm 5.4100$ \\
$\lambda_{\mathrm{RE}}$ using fixed powers (Mbits/joule) & 302.6109 & 2.0442 & 40.2215 & $57.5342 \pm 2.4959$ \\
\hline
\end{tabular}

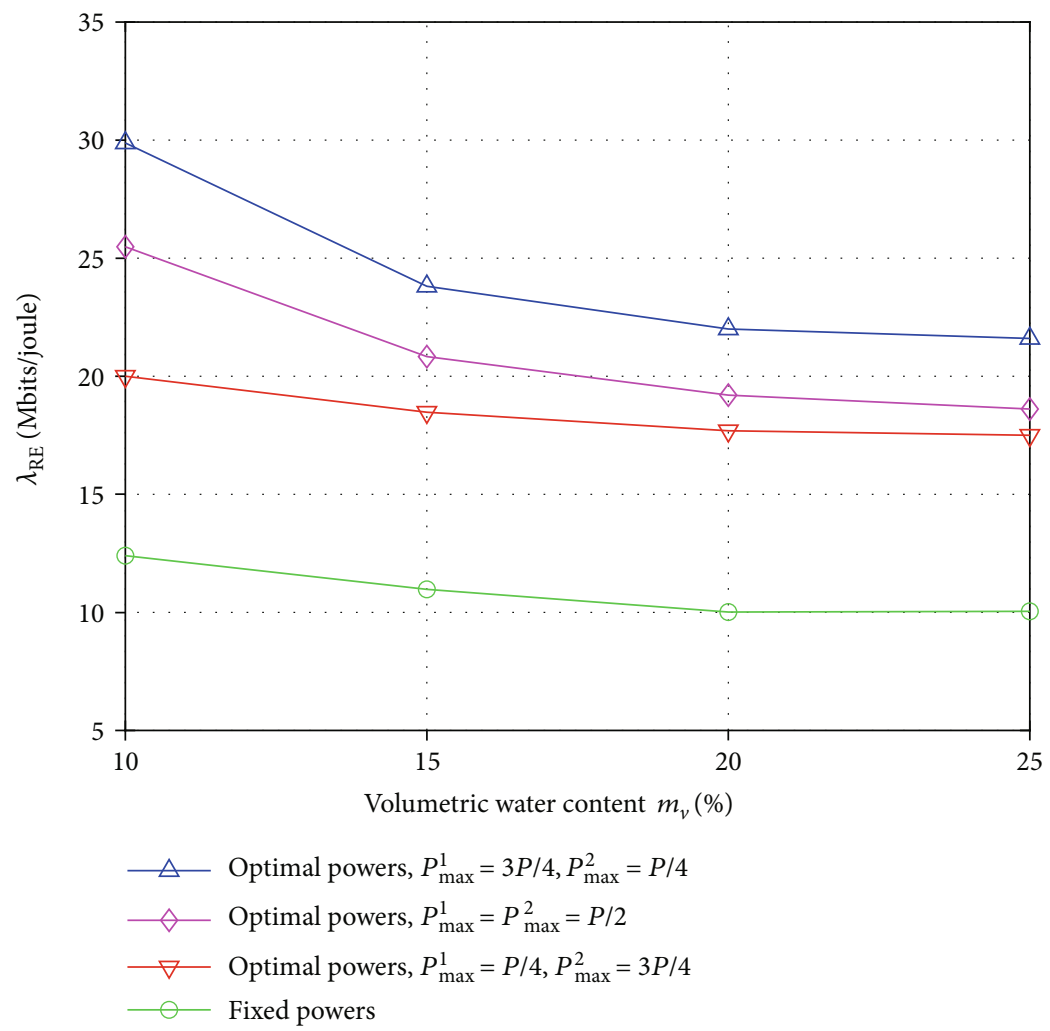

FIGURE 10: Resource efficiency of the proposed power allocation scheme when the volumetric water content (VWC) $m_{v}$ variates among transmissions for $T=2$ with $d_{S G}=0.4 \mathrm{~m}, d_{R G}=0.2 \mathrm{~m}, \mathbf{P}=3 \mathrm{~V}, P_{\max }=50 \mathrm{mw}$, and $W=300 \mathrm{MHz}$.

surface. Obviously, the UG2AG path loss $L_{R A}$ increases when $d_{R G}$ increases while the UG2UG path loss $L_{S R}$ decreases when $d_{R G}$ increases. We notice that $\lambda_{\mathrm{RE}}$ is high when $R$ is buried close to the surface due to the good quality of the UG2AG link. When the relay is in middle location between $S$ and the ground surface, $\lambda_{\mathrm{RE}}$ is low due to the increase of $L_{R A}$, i.e, poor quality of UG2AG link. Moreover, $\lambda_{\mathrm{RE}}$ increases again when $R$ is closer to $S$ although the high value of the path loss $L_{R A}$. Indeed, the enhancement of the UG2UG link predominates the deterioration of the UG2AG link and conducts the enhancement of $\lambda_{\mathrm{RE}}$. This is very important in the topology design of WUSNs where relay nodes are deployed. Placing the relay close to the sensor else to the ground surface is beneficial to the $\lambda_{\mathrm{RE}}$ performance.

Figure 9 shows the resource efficiency $\lambda_{\mathrm{RE}}$ performance of the proposed node power optimization scheme compared to the conventional WUSN scheme with fixed node powers versus the VWC $m_{v}$. We consider $d_{S G}=0.6 \mathrm{~m}, d_{R G}=0.1 \mathrm{~m}$, $\mathbf{P}=3 \mathrm{~V}, P_{\max }=50 \mathrm{mw}$, and $W=300 \mathrm{MHz}$. As shown in the figure, the proposed scheme with optimal node powers ameliorates the $\lambda_{\mathrm{RE}}$ performance compared to the conventional WUSN scheme for the same soil condition in terms of water content. Moreover, the resource efficiency $\lambda_{\mathrm{RE}}$ significantly decreases with higher water content in soil due to the significant UG2UG path loss increase when $m_{v}$ increases, as proven in [4].

In Figure 10, we study the effect of water content $m_{v}$ variation among different transmissions on the resource efficiency $\lambda_{\mathrm{RE}}$. We focus, here, to only two successive transmissions performed through the link $S-R-A$. Then, $T=2$. We assume that $m_{v}$ equals $5 \%$ at the first transmission and $m_{v}$ is changed to a value in the range $(10 \%, 25 \%)$ at the second transmission. We consider $d_{S G}=0.4 \mathrm{~m}, d_{R G}=0.2 \mathrm{~m}, \mathbf{P}=3 \mathrm{~V}, P_{\max }=50 \mathrm{mw}$, and $W=300 \mathrm{MHz}$. As mentioned in Section 4.4, we propose to allocate unequal maximum powers $P_{\max }^{1}=\delta^{1} \mathbf{P}$ and $P_{\max }^{2}=\delta^{2} \mathbf{P}=\left(1-\delta^{1}\right) \mathbf{P}$, where $\delta^{t} \in[0,1], t \in\{1,2\}$, allowed to the first and to the second transmission, respectively.

Figure 10 illustrates the resource efficiency $\lambda_{\mathrm{RE}}$ performance of the proposed node power optimization scheme for the following 4 cases:

(i) $\delta^{1}=3 / 4$, i.e., $P_{\max }^{1}=3 \mathbf{P} / 4$ and $P_{\max }^{2}=\mathbf{P} / 4$ (blue curve)

(ii) $\delta^{1}=1 / 2$, i.e., $P_{\max }^{1}=P_{\max }^{2}=\mathbf{P} / 2$ (pink curve) 
(iii) $\delta^{1}=1 / 4$, i.e., $P_{\max }^{1}=\mathbf{P} / 4$ and $P_{\max }^{2}=3 \mathbf{P} / 4$ (red curve)

(iv) Conventional WUSN scheme with fixed node powers (green curve)

We remark that the proposed node power optimization scheme when $P_{\max }^{1}=P_{\max }^{2}=\mathbf{P} / 2$ outperforms the conventional WUSN scheme with fixed node powers when $m_{v}$ is changed between transmissions. Furthermore, the redistribution of the maximum powers offers the best $\lambda_{\mathrm{RE}}$ performance when $P_{\max }^{1}=3 \mathbf{P} / 4$ and $P_{\max }^{2}=\mathbf{P} / 4$. This motivates us to allocate more power to the first transmission where $m_{v}$ is relatively low and to not spend a lot of power in the second transmission where $m_{v}$ is high. The novel proposed maximum power allocation successfully divides the available battery capacity $\mathbf{P}$ on different transmissions according to the soil condition in each transmission and additionally ameliorates the total $\lambda_{\mathrm{RE}}$. It should be noted that the adjustment of the distribution of the power budget among transmissions should be emphasized in future works.

The deployment of sensor nodes in WUSNs may be restricted in some situations since it depends highly on the application and the environmental conditions. For agricultural application, authors in [4] investigate that the increase of VWC more than $25 \%$ results the interruption of the UG2UG communication for some particular soil type where the capacity to hold the bound water is low. Consequently, in case of a rainfall, the UG2UG communication can remain interrupted for several days depending on the type of the soil. For underground mine application, UG2UG communications may break down in case of an incident, i.e., rock fall or explosion. We notice that the extended periods of communication interruption in such cases should be taken into account in the proposed power allocation algorithm and, generally, in the design of any new protocols.

\section{Conclusions}

In this paper, we studied the problem of sensor and relay power allocation in WUSNs. This is aimed at maximizing the RE, which represents the EE-SE tradeoff, of all allowed transmissions under node battery capacity constraint. The metaheuristic algorithm SSA is used to find optimal node powers from an interval between a minimum and a maximum allowed value. Both invariable and dynamic soil properties among node transmissions are considered. Simulations showed that the proposed scheme provided a better RE for the same resource, in terms of power cost and system bandwidth, compared to the conventional WUSN scheme where fixed maximum powers are used. Also, the proposed scheme efficiently increased the number of transmissions that can be performed through a link and additionally extended the network lifetime. The optimal relay node placement according to the RE maximization is also discussed. For dynamic underground system, we found, through simulations, that allocating more power to the transmission where the water content is low, compared to the second transmission where the water content is high, is the best power distribution maximizing the RE performance.

\section{Data Availability}

No data were used to support this study.

\section{Conflicts of Interest}

The authors declare no conflict of interest.

\section{Acknowledgments}

This project was supported by the Deanship of Scientific Research at Prince Sattam Bin Abdulaziz University under the research project 2019/01/11460.

\section{References}

[1] I. F. Akyildiz and E. P. Stuntebeck, "Wireless underground sensor networks: research challenges," Ad Hoc Networks, vol. 4, no. 6, pp. 669-686, 2006.

[2] M. Yigit, H. U. Yildiz, S. Kurt, B. Tavli, and V. C. Gungor, “A survey on packet size optimization for terrestrial, underwater, underground, and body area sensor networks," International Journal of Communication Systems, vol. 31, no. 11, article e3572, 2018.

[3] S. S. Muhammad, X. Wan, Y. Yang, K. Farzana, and W. A. Mohammad, "Wireless underground sensor networks," International Journal of Performability Engineering, vol. 15, no. 11, pp. 3042-3051, 2019.

[4] M. C. Vuran and I. F. Akyildiz, "Channel model and analysis for wireless underground sensor networks in soil medium," Physical Communication, vol. 3, no. 4, pp. 245-254, 2010.

[5] X. Dong and M. C. Vuran, "Environment aware connectivity for wireless underground sensor networks," in IEEE Proceedings (INFOCOM), pp. 674-682, Turin, Italy, April 2013.

[6] H. T. H. Trang, L. T. Dung, and S. O. Hwang, "Connectivity analysis of underground sensors in wireless underground sensor networks," Ad Hoc Networks, vol. 71, pp. 104-116, 2018.

[7] X. Dong, M. C. Vuran, and S. Irmak, “Autonomous precision agriculture through integration of wireless underground sensor networks with center pivot irrigation systems," Ad Hoc Networks, vol. 11, no. 7, pp. 1975-1987, 2013.

[8] D. Wu, D. Chatzigeorgiou, K. Youcef-Toumi, S. Mekid, and R. Ben-Mansour, "Channel-aware relay node placement in wireless sensor networks for pipeline inspection," IEEE Transactions on Wireless Communications, vol. 13, no. 7, pp. 35103523, 2014.

[9] B. Yuan, H. Chen, and X. Yao, "Optimal relay placement for lifetime maximization in wireless underground sensor networks," Information Sciences, vol. 418-419, pp. 463-479, 2017.

[10] Y. Bao, L. Zhao, Y. Hu, C. Tang, and Z. Aijuan, "Virtual movement of relay nodes for two-tier wireless sensor networks in tunnels," International Journal of Distributed Sensor Networks, vol. 13, no. 1, 2017.

[11] R. Sharma and S. Prakash, "Enhancement of relay nodes communication approach in WSN-IoT for underground coal mine," Journal of Information and Optimization Sciences, vol. 41, no. 2, pp. 521-531, 2020.

[12] J. Tang, D. K. C. So, E. Alsusa, and K. A. Hamdi, "Resource efficiency: a new paradigm on energy efficiency and spectral 
efficiency tradeoff," IEEE Transactions on Wireless Communications, vol. 13, no. 8, pp. 4656-4669, 2014.

[13] J. Tang, D. K. C. So, E. Alsusa, K. A. Hamdi, and A. Shojaeifard, "On the energy efficiency-spectral efficiency tradeoff in MIMO-OFDMA broadcast channels," IEEE Transactions on Vehicular Technology, vol. 65, no. 7, pp. 5185-5199, 2016.

[14] H. Pervaiz, L. Musavian, Qiang Ni, and Zhiguo Ding, "Energy and spectrum efficient transmission techniques under QoS constraints toward green heterogeneous networks," IEEE Access, vol. 3, pp. 1655-1671, 2015.

[15] O. Amin, E. Bedeer, M. H. Ahmed, and O. A. Dobre, "Energy efficiency-spectral efficiency tradeoff: a multiobjective optimization approach," IEEE Transactions on Vehicular Technology, vol. 65, no. 4, pp. 1975-1981, 2016.

[16] G. Yenihayat and E. Karaşan, "Downlink data rate, energy and spectral efficiency distribution in heterogeneous networks with cell-edge located small cells," Wireless Networks, vol. 26, no. 4, pp. 2595-2608, 2020.

[17] S. Kisseleff, I. F. Akyildiz, and W. H. Gerstacker, "Throughput of the magnetic induction based wireless underground sensor networks: key optimization techniques," IEEE Transactions on Communications, vol. 62, no. 12, pp. 4426-4439, 2014.

[18] G. Liu, Z. Wang, and T. Jiang, "QoS-aware throughput maximization in wireless powered underground sensor networks," IEEE Transactions on Communications, vol. 64, no. 11, pp. 4776-4789, 2016.

[19] G. Liu, Z. Sun, and T. Jiang, "Joint time and energy allocation for QoS-aware throughput maximization in MIMO-based wireless powered underground sensor networks," IEEE Transactions on Communications, vol. 67, no. 2, pp. 1400-1412, 2019.

[20] H. Xu, Q. Li, J. Wang, G. Luo, C. Zhu, and W. Sun, “An optimization routing algorithm for green communication in underground mines," Sensors, vol. 18, no. 6, article 1950, 2018.

[21] A. Halber and D. Chakravarty, "Wireless relay placement optimization in underground room and pillar mines," Mathematical Modelling of Engineering Problems, vol. 5, no. 2, pp. 67-75, 2018.

[22] C. Ju, Y. Gao, A. K. Sangaiah, and G. J. Kim, “A PSO based energy efficient coverage control algorithm for wireless sensor networks," Computers, Materials Continua, vol. 56, no. 3, pp. 433-446, 2018.

[23] S. Ebrahimi Mood and M. M. Javidi, "Energy-efficient clustering method for wireless sensor networks using modified gravitational search algorithm," Evolving Systems, vol. 11, no. 4, pp. 575-587, 2020.

[24] A. Zahedi and F. Parma, “An energy-aware trust-based routing algorithm using gravitational search approach in wireless sensor networks," Peer-to-Peer Networking and Applications, vol. 12, no. 1, pp. 167-176, 2019.

[25] P. Singh Mann and S. Singh, "Energy efficient clustering protocol based on improved metaheuristic in wireless sensor networks," Journal of Network and Computer Applications, vol. 83, pp. 40-52, 2017.

[26] R. Kumar and D. Kumar, "Multi-objective fractional artificial bee colony algorithm to energy aware routing protocol in wireless sensor network," Wireless Networks, vol. 22, no. 5, pp. 1461-1474, 2016.

[27] P. Li, H. Nie, L. Qiu, and R. Wang, "Energy optimization of ant colony algorithm in wireless sensor network," Interna- tional Journal of Distributed Sensor Networks, vol. 13, no. 4, 2017.

[28] S. Mirjalili, A. H. Gandomi, S. Z. Mirjalili, S. Saremi, H. Faris, and S. M. Mirjalili, "Salp Swarm Algorithm: a bio-inspired optimizer for engineering design problems," Advances in Engineering Software, vol. 114, pp. 163-191, 2017.

[29] A. A. Ateya, A. Muthanna, A. Vybornova et al., "Chaotic salp swarm algorithm for SDN multi-controller networks," Engineering Science and Technology, an International Journal, vol. 22, no. 4, pp. 1001-1012, 2019.

[30] E. E. Elattar and S. K. ElSayed, "Probabilistic energy management with emission of renewable micro-grids including storage devices based on efficient salp swarm algorithm," Renewable Energy, vol. 153, pp. 23-35, 2020.

[31] A. Singh and V. Sharma, "Salp swarm algorithm-based model predictive controller for frequency regulation of solar integrated power system," Neural Computing and Applications, vol. 31, no. 12, pp. 8859-8870, 2019.

[32] S. Ahmed, M. Mafarja, H. Faris, and I. Aljarah, "Feature selection using salp swarm algorithm with chaos," in Proceedings of the 2nd International Conference on Intelligent Systems, Metaheuristics \& Swarm Intelligence - ISMSI '18, pp. 65-69, Phuket, Thailand, March 2018.

[33] M. B. Nejad and M. E. Shiri, "A new enhanced learning approach to automatic image classification based on Salp Swarm Algorithm," Computer Systems Science and Engineering, vol. 34, no. 2, pp. 91-100, 2019.

[34] X. Shi, J. Su, Z. Ye, F. Chen, P. Zhang, and F. Lang, “A wireless sensor network node location method based on Salp Swarm Algorithm," in 2019 10th IEEE International Conference on Intelligent Data Acquisition and Advanced Computing Systems: Technology and Applications (IDAACS), pp. 357-361, Metz, France, September 2019.

[35] H. M. Kanoosh, E. H. Houssein, and M. M. Selim, "Salp swarm algorithm for node localization in wireless sensor networks," Journal of Computer Networks and Communications, vol. 2019, Article ID 1028723, 12 pages, 2019.

[36] A. Vinitha, M. S. S. Rukmini, and Dhirajsunehra, "Secure and energy aware multi-hop routing protocol in WSN using Taylor-based hybrid optimization algorithm," Journal of King Saud University-Computer and Information Sciences, 2019.

[37] M. A. Syed and R. Syed, "Weighted Salp Swarm Algorithm and its applications towards optimal sensor deployment," Journal of King Saud University-Computer and Information Sciences, 2019.

[38] H. Guo and B. Ben, "Reinforcement learning-enabled reliable wireless sensor networks in dynamic underground environments," in MILCOM 2019 - 2019 IEEE Military Communications Conference (MILCOM), pp. 646-651, Norfolk, VA, USA, November 2019.

[39] M. C. Dobson, F. T. Ulaby, M. T. Hallikainen, and M. el-rayes, "Microwave dielectric behavior of wet soil-part II: dielectric mixing models," IEEE Transactions on Geoscience and Remote Sensing, vol. GE-23, no. 1, pp. 35-46, 1985.

[40] J. H. Scott, "Electrical and magnetic properties of rock and soil," US Geological Survey, pp. 83-915, 1983.

[41] N. R. Peplinski, F. T. Ulaby, and M. C. Dobson, "Dielectric properties of soils in the $0.3-1.3-\mathrm{GHz}$ range," IEEE Transactions on Geoscience and Remote Sensing, vol. 33, no. 3, pp. 803-807, 1995. 
[42] W. E. Patitz, B. C. Brock, and E. G. Powell, "Measurement of dielectric and magnetic properties of soil," vol. 27, Tech. Rep. 12, Sandia National Labs, 1995.

[43] X. Dong and M. C. Vuran, "Impacts of soil moisture on cognitive radio underground networks," in 2013 First International Black Sea Conference on Communications and Networking (BlackSeaCom), pp. 222-227, Batumi, Georgia, July 2013.

[44] L. Abualigah, M. Shehab, M. Alshinwan, and H. Alabool, "Salp swarm algorithm: a comprehensive survey," Neural Computing and Applications, vol. 32, no. 15, pp. 11195-11215, 2020. 